\title{
Characterization of the holography performance of a Titan 80-300 with high brightness Schottky electron gun and image Cs-corrector at $300 \mathrm{kV}$ acceleration voltage
}

\author{
B. Freitag*, S. Kujawa*, M. Linck***, D. Geiger***, T. Niermann**, M. Lehmann**, H. Lichte*** \\ * FEI Company, Eindhoven, Building AAE, Achtseweg Noord 5, P.O. Box 80066, 5600 KA \\ Eindhoven, The Netherlands \\ ** Technische Universität Berlin, Institut für Optik und Atomare Physik, Straße des 17. Juni 135, \\ 10623 Berlin, Germany \\ *** Technische Universität Dresden, Triebenberg Labor, Zum Triebenberg 50, 01328 Dresden, \\ Germany
}

Holography is a powerful method to determine the atomic structure and magnetic fields /electric potentials of nanomaterials. However, not only the spatial resolution is an important parameter for the quality of the electron hologram: Here, an often neglected figure of merit is the phase detection limit determining the smallest phase difference, which can be discriminated between adjacent reconstructed data points (pixels). The phase detection limit is given by

$$
\delta \varphi=\frac{s n r}{V} \sqrt{\frac{2}{N_{r e c}}},
$$

where $s n r=3$ denotes the desired signal-to-noise ratio of the reconstructed phase, $V$ the interference fringe contrast, and $N_{r e c}$ the number of electrons in the reconstructed pixel. In practice, this can be approximated from the hologram for a strong object by

$$
\delta \varphi=\frac{2}{3} \frac{s n r}{V} \sqrt{\frac{2}{s r N_{p i x} / c r}}
$$

with $s r$ the sampling rate of an interference fringe, $N_{p i x}$ the mean number of digital counts per pixel, and $\mathrm{cr}$ the conversion rate of the CCD-camera.

In order to improve the phase detection limit, both the interference fringe contrast $V$ and the number of recorded electrons $N_{p i x} / \mathrm{cr}$ per pixel have to be increased. Where $V$ is mainly given by the spatial coherence of the electron source and the MTF of the CCD-camera, $N_{p i x} / c r$ is directly connected to the coherent current hence the brightness of the electron source.

The new high brightness gun of FEI has approx. a 5 times higher brightness than a conventional Schottky field emission gun. Mounted on a Titan 80-300 column equipped with an image Cscorrector correcting the spherical aberration of the objective lens, this instrument is ideally suited for electron holography. By variation of the illumination system setup, the best settings for gun lens and spot size are evaluated for high resolution electron holography (tab. 1). The resulting phase detection limit is a tradeoff between fringe contrast and number of electrons, where the latter is dramatically improved (fig. 1) in comparison to the conventional Schottky FEG at approximately the same interference fringe contrast. 
It is noteworthy that the small sampling of the fringe spacing $(\sim 5)$ and the small pixel size of $14 \mu \mathrm{m}$ limits the contrast due to the MTF of the camera (Tab.1 and Fig. 1). Improvements in contrast can be obtained using the extra magnification of a post column energy filter camera.

\begin{tabular}{|l|c|c|c|c|}
\hline Image \# & $\mathbf{1}$ & $\mathbf{2}$ & $\mathbf{3}$ & $\mathbf{4}$ \\
\hline Gun Lens & 2 & 1 & 2 & 3 \\
\hline Spot Size & 2 & 3 & 3 & 3 \\
\hline Mean Counts on Camera $\boldsymbol{N}_{\text {pix }}$ & 9936 & 13034 & 4657 & 2436 \\
\hline Fringe Contrast $\boldsymbol{V}$ & $5.64 \%$ & $2.19 \%$ & $6.07 \%$ & $5.67 \%$ \\
\hline Sampling Rate per Fringe $\boldsymbol{s} \boldsymbol{r}$ & 5.21 & 5.02 & 4.98 & 4.98 \\
\hline Phase Detection Limit $\boldsymbol{\delta} \boldsymbol{\varphi}$ & $2 \pi / 14.4$ & $2 \pi / 6.3$ & $2 \pi / 10.4$ & $2 \pi / 7.1$ \\
\hline
\end{tabular}

Tab.1: Variation of the illumination system setup and the resulting hologram parameters for SubÅngström fringe spacing. Note the extremely high mean counts per pixel stemming from the high brightness of the new Schottky FEG. The phase detection is determined by application of eqn.1. Experimental values: Titan 80-300 with image Cs-corrector, acceleration voltage $300 \mathrm{kV}$, 3condenser lens system active, condenser aperture diameter $150 \mu \mathrm{m}$, nominal magnification $1.4 \mathrm{Mx}$, reduced diffraction lens current of $65 \%$, biprism voltage $420 \mathrm{~V}$, fringe spacing $0.062 \mathrm{~nm}$, Gatan USC 1000 CCD-camera, pixel size $14 \mu \mathrm{m}$, conversion rate 3.9 counts/electron, exposure time $4 \mathrm{~s}$.

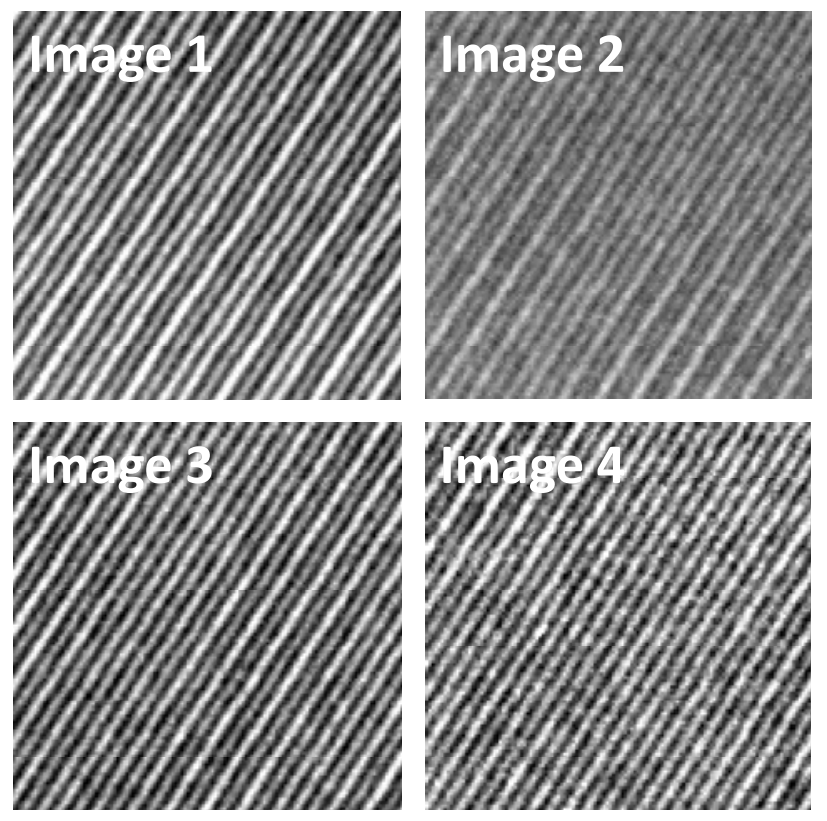

Fig.1: Highly magnified areas of small parts from interference fringes recorded under different illumination system conditions as given in tab.1. Image 1 shows the best signal-to-noise ratio as the result of the tradeoff between interference fringe contrast $V$ and number of recorded electrons $N_{p i x} / c r$ $(\delta \varphi \sim 2 \pi / 14)$. In order to compare the quality of the interference fringes, the dynamic of shown grey values is given by mean counts $N_{p i x} \pm 10 \%$. 